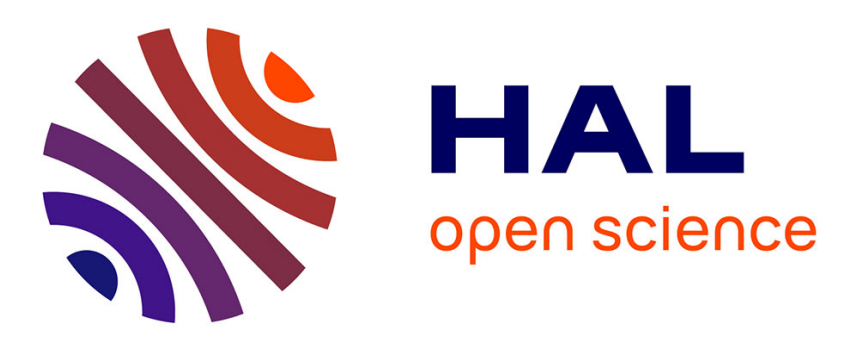

\title{
Optimal feeding strategy for the minimal time problem of a fed-batch bioreactor with mortality rate
}

\author{
Térence Bayen, Francis Mairet, Marc Mazade
}

\section{To cite this version:}

Térence Bayen, Francis Mairet, Marc Mazade. Optimal feeding strategy for the minimal time problem of a fed-batch bioreactor with mortality rate. Optimal Control Applications and Methods, 2015, 36

(1), pp.77-92. 10.1002/oca.2102 . hal-00759058v2

\section{HAL Id: hal-00759058 \\ https://hal.science/hal-00759058v2}

Submitted on 3 Nov 2013

HAL is a multi-disciplinary open access archive for the deposit and dissemination of scientific research documents, whether they are published or not. The documents may come from teaching and research institutions in France or abroad, or from public or private research centers.
L'archive ouverte pluridisciplinaire HAL, est destinée au dépôt et à la diffusion de documents scientifiques de niveau recherche, publiés ou non, émanant des établissements d'enseignement et de recherche français ou étrangers, des laboratoires publics ou privés. 


\title{
Optimal feeding strategy for the minimal time problem of a fed-batch bioreactor with mortality rate
}

\author{
T. Bayen ${ }^{1 *}$, F. Mairet ${ }^{2}$, M. Mazade ${ }^{3}$ \\ 1'INRA-INRIA 'MODEMIC' team, UMR INRA-SupAgro 729 'MISTEA' 2 place Viala 34060 Montpellier \\ 2 INRIA 'BIOCORE' team, Sophia-Antipolis, 2004, route des Lucioles, 06902 Sophia Antipolis Cedex, \\ ${ }^{3}$ Université Montpellier 2, Case courrier 051, 34095 Montpellier cedex 5, France
}

\begin{abstract}
SUMMARY
This paper is devoted to the study of an optimal control problem for a fed-batch bioreactor with one species and one substrate. Our objective is to obtain an optimal feedback control, steering the system in minimal time to a given target defined by conditions on the substrate concentration and the volume of the reactor. The novelty in this work is that a mortality rate for the biomass and hydrolysis of dead biomass are included in the model. The optimal synthesis (optimal feeding strategy) has been obtained by Moreno (1999) when both mortality and hydrolysis are considered negligible. Whenever the model includes these effects, the total mass of the system is no longer conserved, and it is not possible to reduce the dimension of the system. Thanks to the Pontryagin maximum principle and the Hamilton-Jacobi equation we overcome this difficulty and provide an optimal synthesis of the problem in the impulsive framework. Copyright (C) 2010 John Wiley \& Sons, Ltd.

Received ...
\end{abstract}

KEY WORDS: Optimal Control, Minimal Time Problem, Impulsive Control, Pontryagin Maximum Principle, Bioreactor.

\section{INTRODUCTION}

Fed-batch bioreactors are widely used in the industry, in particular for wastewater treatment (see e.g. [1]). The input flow rate in this system is a key control parameter, and finding an adequate feeding strategy can significantly increase process performance.

Our objective is to find an optimal control which steers the system to a given target in minimal time for a reactor with one substrate and one biomass. Following [2,3], we consider a typical target (of interest in wastewater treatment) defined as follows: at the terminal time, the volume has reached the maximal volume of the bioreactor and the substrate concentration is below a value of reference. The novelty in this work is that we assume that the biomass has a mortality rate [4] and that nutrients can be regenerated from a fraction of dead biomass with a given hydrolysis rate (see e.g. [5] for studies of the chemostat model with this phenomena, also called nutrient recycling). Taking into account these effects is a way to introduce uncertainty in the system and is more realistic.

Following [3], the process can be described by a three-dimensional system (representing the substrate and biomass concentrations, and the volume). When mortality and hydrolysis are not present in the model, the system admits a conservation law (the total mass of the system). Thus, the controlled system can be reduced into a two-dimensional affine system with one input (see

\footnotetext{
${ }^{*}$ Correspondence to: Université Montpellier 2, CC 051, 34095 Montpellier cedex 5, France. E-mail: tbayen@ math.univmontp2.fr 
[3]), and one can obtain an optimal control by using the clock form argument (see [6, 7]). This tool is essentially related to planar problems and does not require explicit computations of optimal trajectories (see [8]). When the growth function is of the Monod or the Haldane type (see e.g. $[9,10])$, the optimal synthesis obtained in [3] is as follows:

- For Monod type kinetics, the optimal strategy is called bang-bang (or fill and wait). The first phase consists in filling the reactor to the maximal volume with the maximum input flow rate. The second phase consists in applying a null control until the substrate concentration becomes lower than the reference value.

- For Haldane type kinetics, the optimal strategy is singular (in reference to singular arcs in optimal control theory). The first phase consists in choosing a control which drives the substrate concentration in minimal time to a certain constant value (which corresponds to the substrate concentration maximizing the growth function). The second phase consists in choosing a control which keeps the substrate concentration equal to this value until reaching the maximal volume of the reactor. Finally, the null control is applied as in the second phase of the bang-bang strategy.

These results have been generalized in the impulsive framework in [11] for multi-species and in [12] for growth functions with two local maxima.

In this paper, our objective is to extend the results above to the case where the model includes mortality and hydrolysis coefficients. The fundamental difference with the previous works on the subject is that we cannot reduce the dimension of the system. It turns out that it is no longer possible to make use of the previous arguments (such as the clock form).

One way to tackle this problem is to follow the approach in $[12,11]$ and to consider a more general problem where the input flow rate is considered in the class of impulsive controls. This corresponds to an instantaneous addition of wasted water in the reactor (that is, an instantaneous dilution). From a practical point of view, an impulsive control corresponds to fed-batch reactors for which the input flow is allowed to take very large values: filling becomes fast enough to neglect the biological phenomena (growth and death) during this time.

In this framework, we characterize optimal feeding strategies in a similar way. Our main result is Theorem 3.4 which extends the optimality results of [11] to the case where both mortality and hydrolysis effects are taken into account. We prove that for Monod type kinetics, the optimal strategy is of type bang-bang. When the growth function is of the Haldane type, the singular arc strategy is optimal. The proof of these results relies on explicit computation of the switching function and properties of the adjoint system, which allow excluding extremal trajectories that are not optimal.

The paper is organized as follows. In section 2, the model is presented, and we state some invariance properties of the system. We then introduce the impulsive framework. In section 3, we first neglige the hydrolysis coefficient, and we prove the optimality of the bang-bang strategy for the Monod growth function (via Hamilton-Jacobi equation), and the optimality of the singular arc strategy for the Haldane growth function (see Theorems 3.1 and 3.3). Finally, we cover the case with both mortality and hydrolysis coefficients (Theorem 3.4).

\section{STATEMENT OF THE PROBLEM}

\subsection{Presentation of the model}

We consider the following controlled system describing a perfectly mixed reactor operated in fedbatch with a unit yield coefficient ${ }^{\dagger}$, a mortality rate $k>0$ for the biomass and an hydrolysis rate

${ }^{\dagger}$ This can be achieved through an affine change of variables. 
$k^{\prime}:=\alpha k, 0 \leq \alpha<1:$
$\left\{\begin{array}{l}\dot{x}=\left(\mu(s)-k-\frac{u}{v}\right) x, \\ \dot{s}=\left[-\mu(s)+k^{\prime}\right] x+\frac{u}{v}\left(s_{i n}-s\right), \\ \dot{v}=u .\end{array}\right.$

Here $x$ is the biomass concentration, $s$ the substrate concentration, and $v$ is the volume of water in the tank. If $v_{m}$ is the volume of the tank, the volume $v$ is allowed to take values in $\left(0, v_{m}\right]$. The parameter $s_{i n}>0$ is the input substrate concentration, and the parameter $\alpha$ is less than 1 taking into account that only a fraction of dead biomass can be regenerated into substrate. The control $u$ represents the dilution rate, and it takes values within the set of admissible controls given by:

$$
\mathcal{U}:=\left\{u:[0, \infty) \rightarrow\left[0, u_{m}\right] \mid u(\cdot) \text { meas. }\right\}
$$

where $u_{m}$ represents the maximum value of the dilution rate. In the following, we take $u_{m}=1$ (by time scaling). The optimal control problem that we consider throughout the paper can be stated as follows. First, consider the target $\mathcal{T}$ defined by:

$$
\mathcal{T}:=\mathbb{R}_{+}^{*} \times\left[0, s_{\text {ref }}\right] \times\left\{v_{m}\right\},
$$

where $s_{\text {ref }}$ is a given reference (low) concentration. Given some initial conditions $\xi_{0}:=\left(x_{0}, s_{0}, v_{0}\right)$ for (2.1), our aim is to minimize the time $t(u)$ with respect to $u \in \mathcal{U}$ in order to steer (2.1) from $\xi_{0}$ to the target $\mathcal{T}$ :

$$
\inf _{u \in \mathcal{U}} t(u) \text { s.t. }\left(x_{u}(t(u)), s_{u}(t(u)), v_{u}(t(u))\right) \in \mathcal{T},
$$

where $\left(x_{u}(\cdot), s_{u}(\cdot), v_{u}(\cdot)\right)$ is the unique solution of (2.1) such that $\left(x_{u}(0), s_{u}(0), v_{u}(0)\right)=\xi_{0}$.

We now make more specific assumptions about the system that will ensure the well-posedness of the problem and the existence of optimal controls. First, we may assume without any loss of generality that $u_{m}=1$. The growth function that we consider throughout the paper is either of the Monod or the Haldane type:

- For a growth function $\mu$ of the Monod type, we have: $\mu(s)=\frac{\bar{\mu} s}{k_{1}+s}$.

- For a growth function $\mu$ of the Haldane type, we have: $\mu(s)=\frac{h_{0} s}{h_{2} s^{2}+s+h_{1}}$ where $h_{i}>0$ and the unique maximum of $\mu$ is achieved at $\bar{s}=\sqrt{\frac{h_{1}}{h_{2}}}$.

Next, we assume that $k$ is small enough in order to guarantee that for certain values of substrate concentration, the growth of biomass is possible. More precisely, we require the following assumptions for the growth function.

Hypothesis 2.1

If $\mu$ is of the Monod type, then we assume that $k$ satisfies:

$$
k<\bar{\mu}
$$

In this case, we call $\tilde{s}_{1}$ the unique substrate concentration $s$ satisfying $\mu\left(\tilde{s}_{1}\right)=k^{\prime}$.

Hypothesis 2.2

If $\mu$ is of the Haldane type, then we assume that $k$ satisfies:

$$
k<\mu(\bar{s}) .
$$

In this case, there exist exactly two substrate concentration values $\tilde{s}_{1}^{\prime}$ and $\tilde{s}_{2}^{\prime}$ such that $\tilde{s}_{1}^{\prime}<\bar{s}<\tilde{s}_{2}^{\prime}$ and $\mu\left(\tilde{s}_{1}^{\prime}\right)=\mu\left(\tilde{s}_{2}^{\prime}\right)=k^{\prime}$. In the following, we assume that $\tilde{s}_{2}^{\prime}$ satisfies:

$$
\tilde{s}_{2}^{\prime} \geq s_{i n}
$$

Inequality (2.6) is essential in order to determine an invariant domain for (2.1) as shown in the next Proposition. In fact, if (2.6) is not satisfied, then initial conditions such that $\tilde{s}_{2}^{\prime} \leq s \leq s_{\text {in }}$ would satisfy $\dot{s} \geq 0$ for any control $u$, and the target would not be reachable. 


\section{Proposition 2.1}

(i) In the case where $\mu$ is of the Monod type, the domain

$$
E_{m}:=\mathbb{R}_{+}^{*} \times\left[\tilde{s}_{1}, s_{i n}\right] \times \mathbb{R}_{+}^{*},
$$

is invariant by (2.1).

(ii) In the case where $\mu$ is of the Haldane type, and under assumption (2.6), the set

$$
E_{h}:=\mathbb{R}_{+}^{*} \times\left[\tilde{s}_{1}^{\prime}, s_{i n}\right] \times \mathbb{R}_{+}^{*},
$$

is invariant by (2.1).

Hereafter, when $\alpha=0$ (that is, $k^{\prime}=0$ ), we denote by $E:=\mathbb{R}_{+}^{*} \times\left[0, s_{i n}\right] \times \mathbb{R}_{+}^{*}$ the set given by (2.8). The proof of the Proposition is based on the following lemma (which is a simple consequence of Gronwall's Lemma).

\section{Lemma 2.1}

Consider the ordinary differential equation (ODE):

$$
\dot{y}=f(t, y),
$$

where $f: \mathbb{R} \times \mathbb{R} \rightarrow \mathbb{R}$ is a Caratheodory function local Lipschitz continuous with respect to $y$. Assume that $f(t, 0) \geq 0$ for all $t$. Then, $\mathbb{R}_{+}^{*}$ is invariant by (2.9).

Proof of Proposition 2.1. For a given $u \in \mathcal{U}$, consider a trajectory $(x, s, v)$ solution of (2.1). From Cauchy-Lipschitz Theorem, we have that $x_{0}>0$ implies $x(t)>0$ for all $t$. Now, we can write $\dot{s}=f(t, s)$, where $f(t, s):=\left[-\mu(s)+k^{\prime}\right] x(t)+\frac{u(t)}{v(t)}\left(s_{i n}-s\right)$. So, if $\mu$ is either of the Monod or the Haldane type, then we have $f\left(t, s_{i n}\right) \leq 0$ for all $t$ (recall (2.6) in the Haldane case). Lemma 2.1 implies that we have $s(t) \leq s_{i n}$ for all $t$ provided that $s(0) \leq s_{i n}$. In the case where the growth function is of the Monod type, we have similarly $f\left(t, \tilde{s}_{1}\right)=\frac{u(t)}{v(t)}\left(s_{i n}-\tilde{s}_{1}\right) \geq 0$, so Lemma 2.1 implies the result. We can apply the same argument in the Haldane case which ends the proof.

Now, we discuss some assumptions for the target. In the rest of the paper, we assume that $s_{\text {ref }}$ satisfies the following assumption.

- If $\mu$ is of the Monod type, we assume that $s_{r e f}>\tilde{s}_{1}$.

- If $\mu$ is of the Haldane type, we assume that $s_{r e f}>\tilde{s}_{1}^{\prime}$.

It follows that the target is reachable from any initial condition in $E_{m}$ (in the Monod case) or $E_{h}$ (in the Haldane case). Indeed, a simple way to drive the system to the target is to let $u=1$ until reaching $v_{m}$, and then we take $u=0$ until $s_{\text {ref }}$ (if necessary). When $u=0$, we have that $s(t)$ is strictly decreasing and converges to the equilibrium $\tilde{s}_{1}$ (when $\mu$ is of the Monod type) or $\tilde{s}_{1}^{\prime}$ (when $\mu$ is of the Haldane type). As $s_{r e f}>\tilde{s}_{1}$ (resp. $s_{r e f}>\tilde{s}_{1}^{\prime}$ ) in the Monod case (resp. in the Haldane case), the trajectory necessarily reaches the target in finite time.

\section{Remark 2.1}

If $s_{r e f} \leq \tilde{s}_{1}$ (resp. $s_{r e f} \leq \tilde{s}_{1}^{\prime}$ ) in the Monod case (resp. Haldane case), then we would have $\dot{s}>0$ for $s=\tilde{s}_{1}$ (resp. $s=\tilde{s}_{1}^{\prime}$ ) for any admissible control $u$. As a consequence, the target is not reachable in finite time from a subset of the initial conditions set (in particular if the initial volume is $v_{0}=v_{m}$ ).

The next remark is essential in the analysis of (2.3) and has motivated this work. If $k=0$, the quantity

$$
M:=v\left(x+s-s_{i n}\right)=v_{0}\left(x_{0}+s_{0}-s_{i n}\right),
$$

is conserved along any trajectory of (2.1). It follows that (2.1) can be gathered into a twodimensional system by writing $x=\frac{M}{v}+s_{i n}-s$ :

$$
\left\{\begin{array}{l}
\dot{s}=-\mu(s)\left[\frac{M}{v}+s_{i n}-s\right]+\frac{u}{v}\left(s_{i n}-s\right), \\
\dot{v}=u .
\end{array}\right.
$$


This makes it possible to solve the minimal time problem by using tools which are more specific to planar problems such as Green's Theorem (see $[6,7,8]$ ). The main feature in this work is that the previous reduction is not possible as we have:

$$
\dot{M}=-k(1-\alpha) x v<0,
$$

hence $M$ is strictly decreasing along any trajectory of (2.1). Solving a minimal time problem in the three-dimensional setting is more difficult. We will overcome this difficulty by allowing impulsive controls.

\subsection{Impulsive framework}

We now consider the minimal time problem with an extension of (2.1) allowing impulsive controls, see $[12,11]$. From a practical point of view, this assumption corresponds to a maximum dilution rate $u_{m} \gg \sup _{s \in\left[0, s_{i n}\right]} \mu(s)$. This framework allows us to compute easily the value function corresponding to the different strategies ("fill and wait" and "singular arc" strategies; see Definitions 3.1 and 3.2). Our aim is to prove the following result:

- For the Monod type growth function, the“fill and wait" strategy is optimal (Theorem 3.1).

- For the Haldane type growth function, the "singular arc" strategy is optimal (Theorem 3.3).

The proof of these results relies on the case $\alpha=0$. The case where $\alpha \neq 0$ is considered in the subsection 3.4 and will be a simple consequence of the case $\alpha=0$. Therefore, we consider the initial system (2.1) with $\alpha=0$ and an additional control $r$ which plays the role of an impulsive control.

$$
\left\{\begin{array}{l}
\dot{x}=\left(r[\mu(s)-k]-\frac{u}{v}\right) x, \\
\dot{s}=-r \mu(s) x+\frac{u}{v}\left(s_{i n}-s\right), \\
\dot{v}=u
\end{array}\right.
$$

The system (2.13) is obtained by a reparametrization of the time in (2.1) when replacing $u$ by an impulsive control. More details can be found in [11, 13, 14]. For simplicity, the new time in (2.13) has still been denoted by $t$. The set of admissible controls is defined as follows (the subscript $i$ is for impulsive):

$$
\mathcal{U}_{i}:=\{\mathbf{u}=(r, u):[0, \infty[\rightarrow \Omega \mid \text { meas. }\},
$$

where $\Omega:=(\{0,1\} \times[0,1]) \backslash\{(0,0)\}$. The control $u$ is the input flow rate as in (2.1) and $r$ represents an impulsive control. An instantaneous addition of volume $v_{+}-v_{-}$(i.e. a jump from volume $v_{-}$to volume $v_{+}$) is achieved by taking $r=0$ on some interval of time $\left[\tau_{-}, \tau_{+}\right]$for system (2.1), and any measurable control $u$ satisfying the condition:

$$
\int_{\tau_{-}}^{\tau_{+}} u(t) d t=v_{+}-v_{-}
$$

(see [11] for more details). In particular, there is no uniqueness of $u$ as long as integral (2.14) is equal to $v_{+}-v_{-}$. An addition of volume $v_{+}-v_{-}$corresponds to a dilution of the substrate and the biomass:

$$
s_{+}=\frac{v_{-}}{v_{+}} s_{-}+\left(1-\frac{v_{-}}{v_{+}}\right) s_{i n}, x_{+}=\frac{v_{-}}{v_{+}} x_{-},
$$

where $s_{-}, x_{-}$are the concentrations before dilution, and $s_{+}, x_{+}$the ones after dilution. Hereafter, we also say that the system has an impulse whenever $r=0$ on some time interval. Thus, a phase where $u=u_{m}$ from a volume $v_{0}$ to $v_{1}$ is represented in the impulsive framework by a jump of the volume from $v_{0}$ to $v_{1}$ (with $r=0$ ), neglecting the biological phenomena during this time interval.

For $\xi=(s, x, v) \in E$ and a control $\mathbf{u} \in \mathcal{U}_{\mathbf{i}}$, let $t_{\xi}(\mathbf{u})$ be the first entry time into the target $\mathcal{T}$. In the impulsive framework, the minimal time problem becomes:

$$
\inf _{\mathbf{u} \in \mathcal{U}_{\mathbf{i}}} \int_{0}^{t_{\xi_{0}}(\mathbf{u})} r(t) d t, \text { s.t. }\left(x\left(t_{\xi_{0}}(\mathbf{u})\right), s\left(t_{\xi_{0}}(\mathbf{u})\right), v\left(t_{\xi_{0}}(\mathbf{u})\right)\right) \in \mathcal{T},
$$


In other words, the time $t(u)$ in (2.3) is changed into the $L^{1}$-norm of the control $r$ (see e.g. [11] for more details on the reparametrization of the minimal time problem with impulsive control). As $\Omega$ is not convex, we apply the Pontryagin maximum principle with control in the set $\Omega^{\prime}:=$ $[0,1] \times[0,1] \backslash\{(0,0)\}$. We will see in sections 3.2 and 3.3 that an optimal feedback control $\mathbf{u}$ satisfies $r \in\{0,1\}$.

\section{OPTIMALITY RESULTS FOR THE IMPULSIVE SYSTEM}

\subsection{Pontryagin maximum principle}

In this part, we apply the Pontryagin principle (PMP) on the impulsive system which gives necessary conditions on optimal trajectories. The Hamiltonian $H:=H\left(x, s, v, \lambda_{x}, \lambda_{s}, \lambda_{v}, \lambda_{0}, r, u\right)$ associated to the system is:

$$
H:=r\left[\left(\lambda_{x}-\lambda_{s}\right) \mu(s) x-k x \lambda_{x}+\lambda_{0}\right]+u\left[\lambda_{v}+\frac{\lambda_{s}\left(s_{i n}-s\right)-\lambda_{x} x}{v}\right] .
$$

Let $\mathbf{u}$ an optimal control and $\xi:=(x, s, v)$ its associated trajectory. Then, there exists $t_{f}>0$, $\lambda_{0} \leq 0$ and $\lambda=\left(\lambda_{x}, \lambda_{s}, \lambda_{v}\right):\left[0, t_{f}\right] \rightarrow \mathbb{R}^{3}$ such that $\left(\lambda_{0}, \lambda(\cdot)\right) \neq 0, \lambda$ satisfies the adjoint equation $\dot{\lambda}=-\frac{\partial H}{\partial \xi}\left(\xi, \lambda, \lambda_{0}, \mathbf{u}\right)$ for a.e. $t \in\left[0, t_{f}\right]$, that is:

$$
\left\{\begin{array}{l}
\dot{\lambda}_{x}=-r\left(\lambda_{x}-\lambda_{s}\right) \mu(s)+r k \lambda_{x}+\frac{u}{v} \lambda_{x} \\
\dot{\lambda}_{s}=-r\left(\lambda_{x}-\lambda_{s}\right) x \mu^{\prime}(s)+\frac{u}{v} \lambda_{s} \\
\dot{\lambda}_{v}=\frac{\left(s_{i n}-s\right) \lambda_{s}-x \lambda_{x}}{v^{2}} u
\end{array}\right.
$$

and we have the maximization condition:

$$
\mathbf{u}(t) \in \operatorname{argmax}_{\mathbf{v} \in \boldsymbol{\Omega}^{\prime}} H\left(\xi(t), \lambda(t), \lambda_{0}, \mathbf{v}\right),
$$

for a.e. $t \in\left[0, t_{f}\right]$. Finally as $x\left(t_{f}\right)$ is free, we have the transversality condition

$$
\lambda_{x}\left(t_{f}\right)=0 .
$$

An extremal trajectory is a quadruplet $\left(\xi(\cdot), \lambda(\cdot), \mathbf{u}(\cdot), \mathbf{t}_{\mathbf{f}}\right)$ satisfying (2.13)-(3.2)-(3.3). We assume in the following that optimal trajectories are normal trajectories, that is $\lambda_{0} \neq 0$, hence we take $\lambda_{0}=-1$ (the fact that $\lambda_{0}$ cannot be zero will be discussed in the two next subsections). As we deal with a minimal time problem, the Hamiltonian is zero along an extremal trajectory:

$$
H=r\left[\left(\lambda_{x}-\lambda_{s}\right) \mu(s) x-k x \lambda_{x}-1\right]+u\left[\lambda_{v}+\frac{\left(s_{i n}-s\right) \lambda_{s}-x \lambda_{x}}{v}\right]=0,
$$

Let $\phi_{1}$ (resp. $\phi_{2}$ ) the switching function associated to the control $r$ (resp. $u$ ):

$$
\left\{\begin{array}{l}
\phi_{1}:=\left(\lambda_{x}-\lambda_{s}\right) \mu(s) x-k x \lambda_{x}-1, \\
\phi_{2}:=\lambda_{v}+\frac{\left(s_{i n}-s\right) \lambda_{s}-x \lambda_{x}}{v} .
\end{array}\right.
$$

The value of an extremal control is given by the sign of $\phi_{1}$ and $\phi_{2}$. For a.e. $t \in\left[0, t_{f}\right]$, we have

$$
\left\{\begin{array}{l}
\phi_{1} \leq 0 \text { and } \phi_{2}=0 \Longrightarrow r=0, \\
\phi_{2} \leq 0 \text { and } \phi_{1}=0 \Longrightarrow u=0,
\end{array}\right.
$$

and we have also:

$$
r(t) \phi_{1}(t)+u(t) \phi_{2}(t)=0,
$$

for a.e. $t \in\left[0, t_{f}\right]$, hence $\phi_{1}$ and $\phi_{2}$ are always negative. When $u=0$ on some time interval, we can take without loss of generality $r=1$ as $(r, u) \neq(0,0)$ (see [12]). When $\phi_{1}=\phi_{2}=0$ on some time 
interval, then, we say that the trajectory has a singular arc. By differentiating with respect to $t$, we obtain:

$$
\left\{\begin{array}{l}
\dot{\phi}_{1}=-u \psi, \\
\dot{\phi}_{2}=r \psi,
\end{array}\right.
$$

where:

$$
\psi:=\frac{x\left(s_{i n}-s\right)}{v}\left(\lambda_{s}-\lambda_{x}\right) \mu^{\prime}(s)
$$

When the derivative of the growth function $\mu$ admits a zero (typically in the case where $\mu$ is of the Haldane type), an optimal control can be singular. The following lemma shows that the characterization of singular arcs is essentially the same as the problem with $k=0$ (see section 3.1).

Lemma 3.1

Let $I=\left[t_{1}, t_{2}\right]$ a singular arc. Then, we have $s(t)=\bar{s}$ for $t \in\left[t_{1}, t_{2}\right]$.

Proof

We have $\phi_{1}(t)=\phi_{2}(t)=0$ for all $t \in I$. By differentiating, we obtain $\left(\lambda_{s}(t)-\lambda_{x}(t)\right) \mu^{\prime}(s(t))=0$ for all $t \in I$. Let us prove that $\lambda_{s}-\lambda_{x}$ does not vanish on some time interval $J:=\left[t_{1}^{\prime}, t_{2}^{\prime}\right]$. Otherwise, we would have $\lambda_{s}(t)-\lambda_{x}(t)=\dot{\lambda}_{s}-\dot{\lambda}_{x}(t)=0$ for all $t \in J$. This condition together with the adjoint system implies that $\lambda_{x}(t)=0$ for all $t \in J$. On the other hand, the expression of the Hamiltonian along the singular arc yields that $-k x \lambda_{x}+1=0$ contradicting the fact that $\lambda_{x}$ is vanishing on $J$. Now, by the continuity property of $\lambda_{s}-\lambda_{x}$ and a similar argument, we can prove that $\lambda_{s}-\lambda_{x}$ has a finite number of zeros. Consequently, we have $\mu^{\prime}(s(t))=0$ for all $t \in I$, which proves the Lemma.

To study properties of singular arcs, we define

$$
\alpha:=\frac{\mu(\bar{s})}{s_{i n}-\bar{s}}, \beta:=\mu(\bar{s})-k>0, \bar{x}:=\left(s_{i n}-\bar{s}\right)\left[1-\frac{k}{\mu(\bar{s})}\right] .
$$

\section{Proposition 3.1}

Let us consider a singular arc with $r=1$ on some time interval $\left[t_{0}, t_{1}\right]$ starting at some point $\left(x_{0}, \bar{s}, v_{0}\right)$. Then, the biomass concentration, the singular control $u_{s}$, and the time $t\left(v, x_{0}, v_{0}\right)$ to steer (2.13) from a volume $v_{0}$ to $v$ are given by:

$$
x(v)=\frac{v_{0}}{v} x_{0}+\left[1-\frac{v_{0}}{v}\right] \bar{x}, u_{s}(v)=\alpha x v, t\left(v, x_{0}, v_{0}\right)=t_{0}+\frac{1}{\beta} \ln \left(\frac{x_{0} v_{0}+\bar{x}\left[v-v_{0}\right]}{x_{0} v_{0}}\right) .
$$

Proof

The value of the singular control is straightforward using $\dot{s}=0$ along the singular arc. Let $\rho:=$ $x v$. By differentiating, we have $\dot{\rho}=\beta \rho$ which gives $x(t) v(t)=x_{0} v_{0} e^{\beta\left(t-t_{0}\right)}$. Now we have $\dot{v}=$ $\alpha x v=\alpha x_{0} v_{0} e^{\beta\left(t-t_{0}\right)}$, and by integrating, we obtain $v=v_{0}+\frac{x_{0}}{\bar{x}} v_{0} e^{\beta\left(t-t_{0}\right)}-\frac{x_{0}}{\bar{x}} v_{0}$. Combining this expression with the one of $\rho$ gives the desired expression of $x$. Finally, the expression of $\rho$ gives that $t\left(v, x_{0}, v_{0}\right)-t_{0}=\frac{1}{\beta} \ln \left(\frac{x v}{x_{0} v_{0}}\right)$, and we find the desired expression of $t\left(v, x_{0}, v_{0}\right)$ by replacing $x$ by its expression.

Next, we assume the following condition that will ensure the controllability of the singular arc with $r=1$ for the problem with mortality (see also [2, 12]):

Hypothesis 3.1

Initial conditions in $E$ are such that:

$$
\mu(\bar{s})\left[\frac{M_{0}}{s_{i n}-\bar{s}}+v_{m}\right] \leq u_{m}=1,
$$

where $M_{0}=v_{0}\left(x_{0}+s_{0}-s_{i n}\right)$. 
Recall that along a trajectory, we have $M=v\left(x+s-s_{i n}\right)$, where $M$ is strictly decreasing by (2.12). Together with (3.10), we obtain for $0<v \leq v_{m}$ :

$$
u_{s}(v)=\alpha\left[M+v\left(s_{i n}-s\right)\right] \leq \alpha\left[M_{0}+v_{m}\left(s_{i n}-\bar{s}\right)\right] \leq 1,
$$

where the second inequality follows from Hypothesis 3.1. It follows that this hypothesis guarantees that the singular control satisfies the bound $u_{s} \leq 1$.

\section{Remark 3.1}

(i) For given volume $0<v_{0}<v_{1}$, one can show that the mapping

$$
k \longmapsto \frac{1}{\mu(\bar{s})-k} \ln \left(1+\frac{s_{i n}-\bar{s}}{x_{0}}\left[\frac{v_{1}}{v_{0}}-1\right]\left[1-\frac{k}{\mu(\bar{s})}\right]\right),
$$

is increasing with respect to $k$, therefore the time to steer the system from $v_{0}$ to $v_{1}$ along the singular arc is greater than the one in absence of mortality.

(ii). Given Hypothesis 3.1, we have $u_{s}(v) \in[0,1]$, for all $v \in\left[v_{0}, v_{m}\right]$, which proves that the singular arc is always controllable. This means that for any volume $v \in\left[v_{0}, v_{m}\right]$, the solution of (2.13) with $u=u_{s}(v)$ satisfies $s=\bar{s}$.

We can also compute the time of an $\operatorname{arc} u=0$.

Lemma 3.2

Let us consider a time interval $\left[t_{0}, t_{1}\right]$ where $u=0$ and $r=1$ from $\left(x_{0}, s_{0}, v_{0}\right) \in E$ with $x_{0}>0$ to $\left(x_{1}, s_{1}, v_{0}\right) \in E$ with $0<s_{1}<s_{0}$. Then, we have:

$$
x_{1}=x_{0}+s_{0}-s+k \int_{s_{0}}^{s_{1}} \frac{d \sigma}{\mu(\sigma)}, t_{1}=t_{0}+\int_{s_{0}}^{s_{1}}-\frac{d s}{\mu(s)\left(x_{0}+s_{0}-s+k \int_{s_{0}}^{s} \frac{d \sigma}{\mu(\sigma)}\right)}
$$

Proof

A straightforward computation shows that we have $\frac{d x}{d s}=\frac{k-\mu(s)}{\mu(s)}$, which gives the desired expressions after an integration (recall that if $s_{0}>0$ and $x_{0}>0$, then we have $x(t)>0$ and $s(t)>0$ for all $t$ so that the integrals are well defined).

Also, one can see immediately from (3.12) that the time of an $\operatorname{arc} u=0$ is greater with mortality than when $k=0$.

\subsection{Optimality result for the Monod growth function}

We consider in this section the case where the growth function is of the Monod type and we prove that the strategy "fill and wait" (see Definition 3.1) is optimal for any value of $k>0$.

Let us first prove that $\lambda_{0} \neq 0$. Define the dilution curve $\mathcal{C}_{0}$ which passes through the point $\left(s_{r e f}, v_{m}\right)$ by:

$$
\gamma_{0}(s):=v_{m} \frac{s_{i n}-s_{r e f}}{s_{i n}-s}, s \in\left(0, s_{r e f}\right] .
$$

Also, we define a subset $\tilde{E} \subset E$ by:

$$
\left.\tilde{E}:=\left\{(x, s, v) \in E \mid v \leq v_{m}, v<\gamma_{0}(s)\right)\right\} .
$$

Proposition 3.2

Any optimal trajectory is such that $\lambda_{0} \neq 0$.

Proof

Let $\left(x_{0}, s_{0}, v_{0}\right) \in E$, and consider an optimal trajectory starting at this point and satisfying $\lambda_{0}=0$. If $v_{0} \geq \gamma_{0}\left(s_{0}\right)$, the target $\mathcal{T}$ can be reached by a single impulse (of null cost) on some time interval $\left[0, t_{f}\right]$ (which is necessarily the optimal trajectory), and we have $\phi_{1} \leq 0$ and $\phi_{2}=0$ on this interval. By differentiating, it follows that $\lambda_{s}-\lambda_{x}$ is zero (as $\mu^{\prime} \neq 0$ ) which gives $\lambda_{x}=0$ from the adjoint equation. Now $\phi_{2}=0$ implies that $\lambda_{v}=0$, and we have a contradiction as $\left(\lambda(\cdot), \lambda_{0}\right)$ is non-zero. 
Let us now assume that $v_{0}<\gamma_{0}\left(s_{0}\right)$. There exists a time interval $I:=\left(t_{1}, t_{2}\right)$ where the optimal trajectory is such that $u=0$ and such that either $t_{1}$ or $t_{2}$ is a switching point between an impulse and an $\operatorname{arc} u=0$ (otherwise the trajectory would not reach the target). We have $\phi_{1}=0$ and $\phi_{2} \leq 0$ on $I$, and by differentiating we obtain similarly as in the case above $\lambda_{s}-\lambda_{x}=0$ on $I$. By the adjoint equation, we obtain that $\lambda_{x}=\lambda_{s}=0$ on $I$. Now, at the switching time, $\phi_{2}$ is vanishing, and so is $\lambda_{v}$. It follows that the pair $\left(\lambda(\cdot), \lambda_{0}\right)$ is zero, which contradicts the PMP.

We now make use of the Hamilton-Jacobi equation associated to (2.16) to characterize optimal trajectories for Monod type kinetics. From the expression of the Hamiltonian, the Hamilton-Jacobi equation associated to the problem reads as follows:

$$
\min \left(0,1+x_{0}\left(\mu\left(s_{0}\right)-k\right) \frac{\partial \mathbf{v}}{\partial x_{0}}-x_{0} \mu\left(s_{0}\right) \frac{\partial \mathbf{v}}{\partial s_{0}}\right)+\min \left(0, \frac{\partial \mathbf{v}}{\partial v_{0}}+\frac{s_{\text {in }}-s_{0}}{v_{0}} \frac{\partial \mathbf{v}}{\partial s_{0}}-\frac{x_{0}}{v_{0}} \frac{\partial \mathbf{v}}{\partial x_{0}}\right)=0
$$

where $\left(x_{0}, s_{0}, v_{0}\right) \in E$, together with the boundary condition $\mathbf{v}\left(x_{0}, s_{0}, v_{0}\right)=0$ on the target $\mathcal{T}$. If $\omega: E \rightarrow \mathbb{R}$ is a given function of class $C^{1}$, we define:

$$
\left\{\begin{array}{l}
H_{1}^{\omega}\left(x_{0}, s_{0}, v_{0}\right):=1+x_{0}\left(\mu\left(s_{0}\right)-k\right) \frac{\partial \omega}{\partial x_{0}}\left(x_{0}, s_{0}, v_{0}\right)-x_{0} \mu\left(s_{0}\right) \frac{\partial \omega}{\partial s_{0}}\left(x_{0}, s_{0}, v_{0}\right), \\
H_{2}^{\omega}\left(x_{0}, s_{0}, v_{0}\right):=\frac{\partial \omega}{\partial v_{0}}\left(x_{0}, s_{0}, v_{0}\right)+\frac{s_{i n}-s_{0}}{v_{0}} \frac{\partial \omega}{\partial s_{0}}\left(x_{0}, s_{0}, v_{0}\right)-\frac{x_{0}}{v_{0}} \frac{\partial \omega}{\partial x_{0}}\left(x_{0}, s_{0}, v_{0}\right),
\end{array}\right.
$$

hence (3.14) is equivalent to the two variational inequalities

$$
\left\{\begin{array}{l}
H_{1}^{\mathbf{v}}\left(x_{0}, s_{0}, v_{0}\right) \geq 0 \\
H_{2}^{\mathbf{v}}\left(x_{0}, s_{0}, v_{0}\right) \geq 0
\end{array}\right.
$$

for any $\left(x_{0}, s_{0}, v_{0}\right) \in E$.

\section{Remark 3.2}

We can expect that when $k$ goes to zero, the value function associated to (2.16) converges to the value function associated to the problem with $k=0$. First, this property is not obvious (some arguments can be found in [15]). Second, such a property is not sufficient in order to deduce optimal feedback controls for (2.16) from the case $k=0$.

Consider a point $\left(x_{0}, s_{0}, v_{0}\right)$ and let $\left(x_{0}^{\prime}, s_{0}^{\prime}, v_{m}\right)$ the point which is obtained by an instantaneous dilution until the maximal volume $v_{m}$. Recall from (2.15) that the new biomass and substrate concentrations are given by:

$$
x_{0}^{\prime}=\frac{v_{0}}{v_{m}} x_{0}, s_{0}^{\prime}=\frac{v_{0}}{v_{m}} s_{0}+\left(1-\frac{v_{0}}{v_{m}}\right) s_{i n} .
$$

\section{Definition 3.1}

From any point $\left(x_{0}, s_{0}, v_{0}\right) \in E$, the strategy fill and wait (FW) is $r=0$ until $v=v_{m}$, and then $u=0$ until $s \leq s_{\text {ref }}$ if $s_{0}^{\prime}>s_{\text {ref }}$. The cost $w$ of this strategy is given by:

(i) If $v_{0} \geq \gamma_{0}\left(s_{0}\right)$, then $w\left(x_{0}, s_{0}, v_{0}\right)=0$.

(ii) If $v_{0}<\gamma_{0}\left(s_{0}\right)$, then $w\left(x_{0}, s_{0}, v_{0}\right)=\int_{s_{0}^{\prime}}^{s_{r e f}}-\frac{d s}{\mu(s)\left[x_{0}^{\prime}+s_{0}^{\prime}-s+k \int_{s_{0}^{\prime}}^{s} \frac{d \sigma}{\mu(\sigma)}\right]}$

\section{Lemma 3.3}

The mapping $w$ is continuous on $E$ and of class $C^{1}$ on $\tilde{E}$. Moreover, it satisfies:

$$
H_{1}^{w}\left(x_{0}, s_{0}, v_{0}\right) \geq 0, H_{2}^{w}\left(x_{0}, s_{0}, v_{0}\right)=0 .
$$

Proof

For convenience, we define:

$$
\rho_{\xi_{0}}(s):=x_{0}^{\prime}+\int_{s_{0}^{\prime}}^{s} \frac{k-\mu(\sigma)}{\mu(\sigma)} d \sigma, s_{r e f} \leq s \leq s_{0}^{\prime},
$$


where $\xi_{0}:=\left(x_{0}, s_{0}, v_{0}\right) \in E$. Notice that we have $\rho_{\xi_{0}}(s) \geq x_{0}^{\prime}$ as $s_{r e f} \geq \tilde{s}_{1}$.

Now, one can see that we have $w=0$ on $E \backslash \tilde{E}$, and $w>0$ on $\tilde{E}$. Let $\left(x_{n}, s_{n}, v_{n}\right) \in \tilde{E}$ a sequence of points which converges to a point $\left(x^{0}, s^{0}, v^{0}\right) \in E$ such that $\left(s_{0}, v_{0}\right) \in \mathcal{C}_{0}$, i.e. $v^{0}=\gamma_{0}\left(s^{0}\right)$. We have that the sequence $s_{n}^{\prime}:=\frac{v_{n}}{v_{m}} s_{n}+\left(1-\frac{v_{n}}{v_{m}}\right) s_{i n}$ converges to $s_{\text {ref }}$ and $s \longmapsto \frac{-1}{\mu(s) \rho_{\xi_{0}}(s)}$ is uniformly bounded by $\frac{1}{\mu\left(s_{r e f}\right) x_{0}^{\prime}}$. So, $w\left(x_{n}, s_{n}, v_{n}\right)$ converges to zero, which proves the continuity of $w$ over $\mathcal{C}_{0}$. Moreover, by the regularity property of the integral, the function $w$ is continuous on $\tilde{E}$, which proves the continuity of $w$ in $E$.

Now, take a point $\left(x_{0}, s_{0}, v_{0}\right) \in \tilde{E}$, i.e. $v_{0}<\gamma_{0}\left(s_{0}\right)$. Recall from (3.17) that $x_{0}^{\prime}$ and $s_{0}^{\prime}$ depend on $\left(x_{0}, s_{0}, v_{0}\right)$ in a continuously differentiable fashion. It follows from the definition of $w$ (see Definition 3.1) that it is of class $C^{1}$ on $\tilde{E}$.

By taking the derivative of $w$ with respect to $x_{0}, s_{0}$ and $v_{0}$, we get:

$$
\begin{gathered}
\frac{\partial w}{\partial x_{0}}\left(x_{0}, s_{0}, v_{0}\right)=\frac{v_{0}}{v_{m}} \int_{s_{0}^{\prime}}^{s_{r e f}} \frac{d s}{\mu(s) \rho_{\xi_{0}}^{2}(s)} d s \\
\frac{\partial w}{\partial s_{0}}\left(x_{0}, s_{0}, v_{0}\right)=\frac{v_{0}}{v_{m}} \frac{1}{x_{0}^{\prime} \mu\left(s_{0}^{\prime}\right)}+\frac{v_{0}}{v_{m}}\left(1-\frac{k}{\mu\left(s_{0}^{\prime}\right)}\right) \int_{s_{0}^{\prime}}^{s_{r e f}} \frac{d s}{\mu(s) \rho_{\xi_{0}}^{2}(s)} d s, \\
\frac{\partial w}{\partial v_{0}}\left(x_{0}, s_{0}, v_{0}\right)=\frac{1}{x_{0}^{\prime} \mu\left(s_{0}^{\prime}\right)} \frac{s_{0}-s_{i n}}{v_{m}}+\left(\frac{x_{0}}{v_{m}}-\frac{s_{0}-s_{i n}}{v_{m}} \frac{k-\mu\left(s_{0}^{\prime}\right)}{\mu\left(s_{0}^{\prime}\right)}\right) \int_{s_{0}^{\prime}}^{s_{r e f}} \frac{d s}{\mu(s) \rho_{\xi_{0}}^{2}(s)} d s .
\end{gathered}
$$

It follows that

$$
H_{1}^{w}\left(x_{0}, s_{0}, v_{0}\right)=1-\frac{\mu\left(s_{0}\right)}{\mu\left(s_{0}^{\prime}\right)}+k x_{0}^{\prime}\left(\frac{\mu\left(s_{0}\right)}{\mu\left(s_{0}^{\prime}\right)}-1\right) \int_{s_{0}^{\prime}}^{s_{r e f}} \frac{d s}{\mu(s) \rho_{\xi_{0}}^{2}(s)} d s .
$$

But, one has $\mu\left(s_{0}\right) \leq \mu\left(s_{0}^{\prime}\right)$ as $s_{0} \leq s_{0}^{\prime}$, and the integral above is negative as $s_{0}^{\prime}>s_{\text {ref }}$, hence $H_{1}^{w}\left(x_{0}, s_{0}, v_{0}\right) \geq 0$. Moreover, a direct computation shows that $H_{2}^{w}\left(x_{0}, s_{0}, v_{0}\right)=0$, and the result follows.

\section{Theorem 3.1}

The feedback control law $\mathbf{u}_{F W}$ given by

$$
\mathbf{u}_{F W}\left(s_{0}, x_{0}, v_{0}\right):= \begin{cases}(0, u), & \text { if } v_{0}<v_{m}, \\ (1,0), & \text { if } v_{0}=v_{m} \text { and } s_{0}>s_{r e f},\end{cases}
$$

is optimal, and the value function associated to (2.16) satisfies $\mathbf{v}=w$.

\section{Proof}

Lemma 3.3 allows us to apply Proposition 5.4 of [11] (giving a sufficient condition for a feedback control law to be optimal). First, any solution of (2.13) with the feedback (3.19) is absolutely continuous and reaches the target in finite time. Moreover, the feedback $\mathbf{u}_{F W}$ is such that:

- The function $w$ is continuous over $E$ and of class $C^{1}$ on $\tilde{E}$ (the set of points where $w>0$ ).

- The function $w$ fulfills (3.18) and consequently (3.14), together with the boundary condition $w=0$ on the target $\mathcal{T}$.

- The function $w$ satisfies $r=0$ for any point in $\tilde{E}$ such that $w=0$.

We can conclude that this strategy is optimal. Note that in (3.25), $u$ is any measurable control taking values in $[0,1]$ such that its integral on the period of the dilution is equal to $v_{m}-v_{0}$, see (2.14). 


\subsection{Optimality result for the Haldane growth function}

We assume in this subsection that $\mu$ is of the Haldane type, and that $\bar{s}>s_{r e f}$. We will prove that the singular arc strategy (see Definition 3.2) is optimal for any value of $k$. The Hamilton-Jacobi equation is a direct way to prove optimality if we have a candidate for the value function, which is the case here. Unfortunately, the expression (3.24) provided by this strategy seems delicate to handle in this case. So, we have used the Pontryagin maximum principle, which allows us to exclude extremal trajectories, and to prove the optimality of the singular arc strategy.

First, we can prove similarly as in the case of the Monod type growth function that $\lambda_{0} \neq 0$ (see Proposition 3.2). The next lemma gives properties of the trajectory during an impulse of volume.

\section{Lemma 3.4}

Consider an extremal trajectory starting at some point $\left(x_{0}, s_{0}, v_{0}\right) \in E$ with $v_{0}<v_{m}$. Assume that we have $r=0$ on some time interval $\left[0, t_{1}\right]$ where $t_{1}$ is a switching point. Then, we have:

$$
\left[\lambda_{x}^{0}-\lambda_{s}^{0}\right]\left[\mu\left(s\left(t_{1}\right)\right)-\mu\left(s_{0}\right)\right] \geq 0,
$$

where $\lambda^{0}:=\left(\lambda_{x}^{0}, \lambda_{s}^{0}, \lambda_{v}^{0}\right)$ is the initial adjoint vector.

Proof

One can see that on $\left[0, t_{1}\right]$, we have $\dot{\lambda}_{x}=\frac{\dot{v}}{v} \lambda_{x}, \dot{\lambda}_{s}=\frac{\dot{v}}{v} \lambda_{s}$, thus $\lambda_{x}=\frac{v}{v_{0}} \lambda_{x}^{0}$ and $\lambda_{s}=\frac{v}{v_{0}} \lambda_{s}^{0}$. This gives

$$
\phi_{1}=\left(\lambda_{x}^{0}-\lambda_{s}^{0}\right) x_{0} \mu(s)-1-k x_{0} \lambda_{x}^{0} .
$$

As $r=0$ on the interval $\left[0, t_{1}\right]$, we have $\phi_{1}(0) \leq 0$ and $\phi_{1}\left(t_{1}\right)=0$ (as $t_{1}$ is a switching point). The lemma follows from (3.21).

We now prove that it is not possible to have an impulse from a point in $\left(x_{0}, s_{0}, v_{0}\right) \in E$ with $v_{0}<v_{m}$ and $s_{0}>\bar{s}$ to the maximal volume.

\section{Lemma 3.5}

Assume that an extremal trajectory satisfies $r=0$ from a point $\left(x_{0}, s_{0}, v_{0}\right) \in E$ with $v_{0}<v_{m}$ and $s_{0}>\bar{s}$ until the maximum volume $v_{m}$. Then, the trajectory is not optimal.

\section{Proof}

Suppose that we have $r=0$ until $v_{m}$ and let $t_{1}$ the time where the trajectory reaches the maximal volume. We then have $u=0$ on $\left[t_{1}, t_{f}\right]$ where $t_{f}>t_{1}$ is such that $s\left(t_{f}\right)=s_{\text {ref }}$ (first entry time into the target). We have $\phi_{1}=0$ on the interval $\left[t_{1}, t_{f}\right]$, therefore

$$
\lambda_{x}-\lambda_{s}=\frac{1+k x \lambda_{x}}{\mu(s) x} .
$$

From the adjoint equation, we get that $\dot{\lambda}_{x}=-\frac{1}{x}$, so $\lambda_{x}$ is decreasing, and using (3.4), we obtain that $\lambda_{x} \geq 0$ on $\left[t_{1}, t_{f}\right]$. Consequently, $\lambda_{x}-\lambda_{s}$ is non-negative on $\left[t_{1}, t_{f}\right]$, thus $\lambda_{x}\left(t_{1}\right)-\lambda_{s}\left(t_{1}\right) \geq 0$. By (3.20), and from the fact that $\mu\left(s_{0}\right)-\mu\left(s\left(t_{1}\right)\right)>0$, we obtain

$$
\lambda_{x}^{0}-\lambda_{s}^{0}<0,
$$

where $\lambda_{0}:=\left(\lambda_{x}^{0}, \lambda_{s}^{0}, \lambda_{v}^{0}\right)$ is the initial adjoint vector. Recall from Lemma 3.20 that along the impulse, we have $\lambda_{x}-\lambda_{s}=\frac{v}{v_{0}}\left[\lambda_{x}^{0}-\lambda_{s}^{0}\right]$. It follows that at time $t_{1}$, we have $\lambda_{x}\left(t_{1}\right)-\lambda_{s}\left(t_{1}\right)=$ $\frac{v_{m}}{v_{0}}\left[\lambda_{x}^{0}-\lambda_{s}^{0}\right]<0$, which is a contradiction.

Corollary 3.1

Any extremal trajectory starting at some point $\left(x_{0}, s_{0}, v_{0}\right) \in E$ with $v_{0}<v_{m}, s_{0}>\bar{s}$, and such that $s(t)>\bar{s}$ for all $t$ such that $v(t)<v_{m}$ is not optimal.

Proof

Such a trajectory is necessarily a concatenation of arcs $u=0$ and $r=0$, and it contains a non-trivial impulse to the maximal volume $v_{m}$, and we can use Lemma 3.5 to conclude. 
Similarly, we show that a trajectory which has a switching point from an $\operatorname{arc} u=0$ to an impulse at a substrate concentration strictly greater than $\bar{s}$, is not optimal.

\section{Lemma 3.6}

Let us consider an extremal trajectory starting at some point $\left(x_{0}, s_{0}, v_{0}\right) \in E$ with $v_{0}<v_{m}, s_{0}>\bar{s}$. Assume that it satisfies $u=0$ on $\left[0, t_{0}\right]$ and $r=0$ on $\left[t_{0}, t_{1}\right]$ where $s\left(t_{0}\right)>\bar{s}$. Then, the trajectory is not optimal.

\section{Proof}

As we have $\phi_{2}<0$ on $\left[0, t_{0}\right)$, we get that $\dot{\phi}_{2}\left(t_{0}\right)=\lim _{t \rightarrow t_{0}} \frac{\phi_{2}(t)-\phi_{2}\left(t_{0}\right)}{t-t_{0}} \geq 0$. We obtain from (3.9) that $\dot{\phi}_{2}=\psi$, thus $\lambda_{s}\left(t_{0}\right)-\lambda_{x}\left(t_{0}\right) \leq 0$ (recall that $\mu^{\prime}\left(s\left(t_{0}\right)\right)<0$ as $s\left(t_{0}\right)>\bar{s}$ ). From the impulse at time $t_{0}$ and from Lemma 3.4, we obtain that necessarily $\lambda_{x}\left(t_{0}\right)-\lambda_{s}\left(t_{0}\right)<0$ which is a contradiction.

We now investigate the case where an extremal trajectory has a switching point at a substrate concentration lower than $\bar{s}$ and for a volume value strictly less than $v_{m}$.

\section{Lemma 3.7}

Consider an extremal trajectory starting at some point $\left(x_{0}, s_{0}, v_{0}\right) \in E$ with $v_{0}<v_{m}, s_{0}<\bar{s}$. Assume that it satisfies $u=0$ on $\left[0, t_{0}\right]$ and $r=0$ on $\left[t_{0}, t_{1}\right]$. Then, the trajectory is not optimal.

\section{Proof}

We have $\phi_{2}<0$ on the interval $\left(0, t_{0}\right)$ and $\phi_{2}\left(t_{0}\right)=0$, therefore $\dot{\phi}_{2}\left(t_{0}\right) \geq 0$. On the interval $\left[0, t_{0}\right]$, the switching function $\phi_{2}$ satisfies $\dot{\phi}_{2}=\psi$, therefore we get $\lambda_{s}\left(t_{0}\right)-\lambda_{x}\left(t_{0}\right) \geq 0$. From Lemma 3.4, we obtain that $\lambda_{x}\left(t_{0}\right)-\lambda_{s}\left(t_{0}\right)>0$ (because $\mu$ is increasing on $\left.[0, \bar{s}]\right)$, hence $\lambda_{s}\left(t_{0}\right)-\lambda_{x}\left(t_{0}\right)<0$, which is a contradiction.

Notice that this Lemma implies that it is not possible for an optimal trajectory to cross the singular arc with $u=0$ at a volume $v_{0}<v_{m}$.

We now prove that it is not optimal for a trajectory to leave the singular arc before reaching the maximal volume. Hereafter, $S_{\left[t_{1}, t_{2}\right]}, I_{\left[t_{1}, t_{2}\right]}$, and $N F_{\left[t_{1}, t_{2}\right]}$ denote a singular arc, an arc $r=0$, and an $\operatorname{arc} u=0$ on some time interval $\left[t_{1}, t_{2}\right]$.

\section{Proposition 3.3}

Consider an extremal trajectory starting at some point $\left(x_{0}, \bar{s}, v_{0}\right) \in E$ at time 0 with $v_{0}<v_{m}$ and which contains a singular arc on some time interval $\left[0, t_{1}\right]$. If the trajectory is optimal, then it is singular until the maximal volume.

\section{Proof}

Without any loss of generality, we may assume that the trajectory is singular until the time $t_{1}$ and that $v\left(t_{1}\right)<v_{m}$. From Lemma 3.7, the trajectory cannot switch to $u=0$ at time $t_{1}$, therefore, if it is optimal, we necessarily have that $r=0$ (a dilution) in a right neigbourhood of $t_{1}$. If we have $r=0$ until the maximal volume, we know from Lemma 3.5 that the trajectory is not optimal. Similarly, if the impulse does not reach the maximal volume, but if the extremal trajectory contains a sequence $I_{\left[t_{1}, t_{2}\right]} N F_{\left[t_{2}, t_{3}\right]} I_{\left[t_{3}, t_{4}\right]}$ with $0<t_{1}<t_{2}<t_{3}<t_{4}, v\left(t_{3}\right)<v_{m}$ and $s\left(t_{3}\right)>\bar{s}$, then we know from Lemma 3.6 that the trajectory is not optimal.

We deduce that the extremal trajectory necessarily consists of sequences of singular arcs followed by a dilution $r=0$ and an $\operatorname{arc} u=0$ until $\bar{s}$. This means that there exists $t_{2}>t_{1}$ such that $r=0$ on $\left[t_{1}, t_{2}\right]$ with $s\left(t_{2}\right)>\bar{s}$, and that at time $t_{2}$, we have $u=0$ until the singular arc which is reached at time $t_{3}$. Therefore, the only possibility for the trajectory is to contain a concatenation of sequences of type $S_{\left[0, t_{1}\right]} I_{\left[t_{1}, t_{2}\right]} N F_{\left[t_{2}, t_{3}\right]}$ until reaching the maximal volume $v_{m}$ (by a singular arc from Lemma 3.5).

We now prove that the existence of such a sequence implies a contradiction, which will prove that it is optimal for a trajectory to be singular until the maximal volume. Let $\varphi:=\lambda_{x}-\lambda_{s}$. 


\section{Claim 3.1}

A sequence $I_{\left[t_{1}, t_{2}\right]} N F_{\left[t_{2}, t_{3}\right]}$ such that $s\left(t_{1}\right)=s\left(t_{3}\right)=\bar{s}$ satisfies $\varphi<0$ on $\left[t_{1}, t_{3}\right]$.

Let us prove Claim 3.1. From Lemma 3.4, we have $\varphi\left(t_{1}\right)<0$ and $\varphi\left(t_{2}\right)<0$. Now, as $u=0$ on $\left[t_{2}, t_{3}\right]$, we have $\phi_{1}=0$ and $\varphi \mu(s) x=1+k x \lambda_{x}$ on this interval. Combining with the adjoint equation gives:

$$
\dot{\varphi}=x \mu^{\prime}(s) \varphi-\frac{1}{x} .
$$

Assume that there exists $\tau \leq t_{3}$ such that $\varphi$ is vanishing. We can assume that $\varphi<0$ on $\left[t_{2}, \tau\right)$ so that $\dot{\varphi}(\tau) \geq 0$. On the other hand, (3.22) implies that $\dot{\varphi}(\tau)=-\frac{1}{x(\tau)}<0$, and we have a contradiction, which proves the claim.

\section{Claim 3.2}

If a sequence $S_{\left[t_{3}, t_{4}\right]}$ satisfies $\varphi\left(t_{3}\right)<0$, then we have $\varphi\left(t_{4}\right)<0$.

Let us prove Claim 3.2. On the interval $\left[t_{3}, t_{4}\right]$, we have $\phi_{1}=\phi_{2}=0$ and $\mu^{\prime}(\bar{s})=0$ which gives:

$$
\dot{\varphi}=\frac{u_{s}}{v} \varphi-\frac{1}{x},
$$

where $u_{s}$ is the singular control (recall (3.10)). From (3.23) and Gronwall's Lemma, we obtain that $\varphi\left(t_{3}\right)<0$ implies $\varphi\left(t_{4}\right)<0$, as was to be proved.

To conclude the proof of the Proposition, note that from our assumption, there exists at least one sequence $S_{\left[0, t_{1}\right]} I_{\left[t_{1}, t_{2}\right]} N F_{\left[t_{2}, t_{3}\right]}$ as above. Combining Lemma 3.4, Claims 3.1 and 3.2, yields that $\varphi\left(t_{1}\right)<0, \varphi\left(t_{2}\right)<0$ and $\varphi\left(t_{3}\right)<0$. By repeating this argument on each such sequence if necessary, we obtain that there exists a time $\bar{t}>0$ such that $s(\bar{t})=\bar{s}, v(\bar{t})=v_{m}$, and $\varphi(\bar{t})<0$. Now, the transversality condition at the terminal time implies that

$$
\varphi\left(t_{f}\right)=\frac{1}{\mu\left(s_{r e f}\right) x\left(t_{f}\right)}>0,
$$

which contradicts $\varphi(\bar{t})<0$ and Claim 3.1 (recall that Claim 3.1 together with $\varphi(\bar{t})<0$ implies $\left.\varphi\left(t_{f}\right)<0\right)$. This concludes the proof.

Let $\mathcal{C}_{1}$ the dilution curve which passes through the point $\left(\bar{s}, v_{m}\right)$, and whose equation is given by $\gamma_{1}(s):=v_{m} \frac{s_{i n}-\bar{s}}{s_{i n}-s}$. The singular arc strategy is defined as follows.

\section{Definition 3.2}

Let $\left(x_{0}, s_{0}, v_{0}\right) \in E$.

(i) If $v_{0} \geq \gamma_{1}\left(s_{0}\right)$, the singular arc strategy coincides with the strategy fill and wait.

(ii) If $s_{0} \leq \bar{s}$, and $v_{0}<\gamma_{1}\left(s_{0}\right)$, the singular arc strategy consists of an impulse from $s_{0}$ to $\bar{s}$, followed by a singular arc until reaching $v=v_{m}$ and then an arc $u=0$ until $s_{r e f}$.

(iii) If $s_{0} \geq \bar{s}$, the singular arc strategy consists of an arc $u=0$ until reaching $\bar{s}$, a singular arc until $v=v_{m}$ and then an $\operatorname{arc} u=0$ until $s_{\text {ref }}$.

The singular strategy satisfies the following property.

Theorem 3.2

For any point $\left(x_{0}, s_{0}, v_{0}\right) \in E$, the optimal feeding policy is the singular arc strategy.

\section{Proof}

Let $\left(x_{0}, s_{0}, v_{0}\right) \in E$ with $v_{0}<v_{m}$. First, assume that $s_{0}<\bar{s}$. If, $v_{0}>\gamma_{1}\left(s_{0}\right)$, Lemma 3.7 implies that $r=0$ until $v_{m}$. In this case, the singular arc strategy coincides with the strategy fill and wait. If $v_{0}<\gamma_{1}\left(s_{0}\right)$, Lemma 3.7 implies that $r=0$ until reaching the singular arc. Otherwise, we would have a switching point to an arc $u=0$ at some time $t_{0}$ with $v\left(t_{0}\right)<v_{m}, s\left(t_{0}\right) \leq \bar{s}$. As $v\left(t_{0}\right)<v_{m}$, the trajectory necessary contains a switching point to $r=0$ at some time $t_{1}>t_{0}$, and we can apply Lemma 3.7 to exclude this possibility. Now, Proposition 3.3 implies that the trajectory is singular until $v=v_{m}$. 
Assume now that $s_{0}>\bar{s}$. From corollary 3.1 and Lemma 3.6, we have $u=0$ until the singular arc. From Proposition 3.3, the trajectory remains singular until $v_{m}$, which ends the proof.

We now give the cost associated to this strategy. When $s_{0}<\bar{s}$ and $v_{0}<\gamma_{1}\left(s_{0}\right)$, we call $x_{0}^{\prime}$ the biomass concentration corresponding to a dilution from $s_{0}$ to $\bar{s}, v_{0}^{\prime}$ the new volume, and $x_{0}^{\prime \prime}$ the biomass concentration at the end of the singular arc (at volume $v_{m}$ ):

$$
x_{0}^{\prime}=x_{0} \frac{s_{i n}-\bar{s}}{s_{i n}-s_{0}}, \quad v_{0}^{\prime}=v_{0} \frac{s_{i n}-s_{0}}{s_{i n}-\bar{s}}, \quad x_{0}^{\prime \prime}=\bar{x}+\frac{v_{0}^{\prime}}{v_{m}}\left(x_{0}^{\prime}-\bar{x}\right) .
$$

When $s_{0}>\bar{s}$, we call $\tilde{x}_{0}^{\prime}$ the biomass concentration corresponding to an $\operatorname{arc} u=0$ until $\bar{s}$, and $\tilde{x}_{0}^{\prime \prime}$ the biomass concentration at the end of the singular arc (at volume $v_{m}$ ):

$$
\tilde{x}_{0}^{\prime}=x_{0}+s_{0}-\bar{s}+k \int_{s_{0}}^{\bar{s}} \frac{d \sigma}{\mu(\sigma)}, \tilde{x}_{0}^{\prime \prime}=\bar{x}+\frac{v_{0}}{v_{m}}\left(\tilde{x}_{0}^{\prime}-\bar{x}\right) .
$$

The cost of the singular arc strategy is:

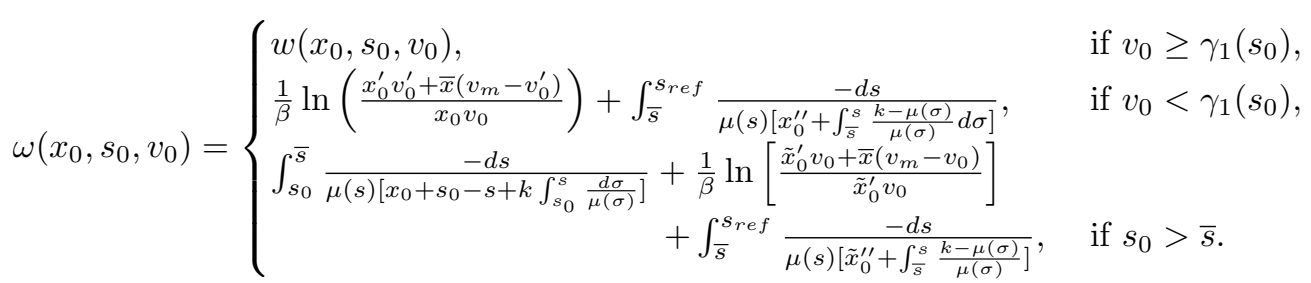

Theorem 3.2 implies the following result.

Theorem 3.3

The feedback control law $\mathbf{u}_{S A}$ given by

$$
\mathbf{u}_{S A}\left(s_{0}, x_{0}, v_{0}\right):= \begin{cases}(0, u), & \text { if } s_{0}<\bar{s}, v_{0}<v_{m}, \\ \left(1, u_{s}(v)\right), & \text { if } s=\bar{s}, v_{0}<v_{m} \\ (1,0), & \text { if } v_{0}=v_{m} \text { or } s_{0}>\bar{s}\end{cases}
$$

is optimal, and the value function associated to (2.16) satisfies $\mathbf{v}=\omega$.

\section{Proof}

The result is a rephrasing of Theorem 3.2 in term of feedback control. Note that in (3.25), $u$ is any measurable control taking values in $[0,1]$ such that its integral on the period of the dilution is equal to $v_{m}-v_{0}$ (see Definition 2.14).

\subsection{Fed-batch bioreactor with mortality and Hydrolysis}

In this section, we investigate the case where both coefficients $k$ and $k^{\prime}$ are non-zero. So, in the impulsive framework, (2.1) becomes:

$$
\left\{\begin{array}{l}
\dot{x}=\left(r[\mu(s)-k]-\frac{u}{v}\right) x, \\
\dot{s}=r\left[-\mu(s)+k^{\prime}\right] x+\frac{u}{v}\left(s_{i n}-s\right), \\
\dot{v}=u
\end{array}\right.
$$

Now, by setting $\nu(s):=\mu(s)-k^{\prime}$, (3.26) becomes:

$$
\left\{\begin{array}{l}
\dot{x}=\left(r\left[\nu(s)-k^{\prime \prime}\right]-\frac{u}{v}\right) x, \\
\dot{s}=-r \nu(s) x+\frac{u}{v}\left(s_{i n}-s\right), \\
\dot{v}=u
\end{array}\right.
$$


where $k^{\prime \prime}=k-k^{\prime}>0$. In view of Proposition 2.1, we are in position to apply the result of Theorem 3.1 and 3.3 to the system (3.27) on the domain $E_{m} \subset E$ (in the case of a Monod type growth function) or $E_{\alpha} \subset E$ (in the case of Haldane type kinetics). Indeed, both domains $E_{m}$ and $E_{\alpha}$ remain invariant for (3.27). Moreover, if $\mu$ is of the Monod type, then $\nu$ is increasing on $\left[\tilde{s}_{1},+\infty\right]$, and if $\mu$ is of the Haldane type, $\nu$ is increasing on $\left[\tilde{s}_{1}^{\prime}, \bar{s}\right]$, and decreasing over $\left[\bar{s}, s_{i n}\right]$. So, we can apply the optimality result on these sets with $\nu$ in place of $\mu$ and state our main result.

\section{Theorem 3.4}

(i) When $\mu$ is of the Monod type, the strategy fill and wait is optimal in the domain $E_{m}$.

(ii) When $\mu$ is of the Haldane type, the singular arc strategy is optimal in the domain $E_{\alpha}$.

\subsection{Numerical simulations}

Finally, we have compared the cost of the optimal strategy with respect to the initial volume value and the mortality coefficient $k$ in order to measure the effect of the mortality on the system. Numerical results are depicted in tables I,II for $k=0,10^{-4}, 10^{-3}$, see also Fig. 1. As expected, the time of the optimal strategy is increasing as a function of $k$ and decreasing with respect to $v_{0}$. When $k$ is small and when the initial volume is close to the target, the time of the optimal trajectory is close to the optimal one with $k=0$. Notice also that even if $k$ is small, the time to reach the target significantly differs with the one with $k=0$ when the initial point is far from the target.

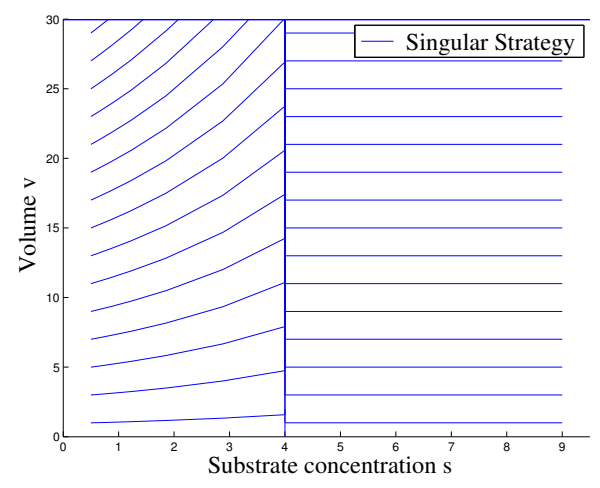

Figure 1. Plot of the projection of the singular arc strategy into the plane $(s, v)$ in the impulsive framework for different initial volume values, with a mortality coefficient $k=10^{-4}$. Parameter values (arbitrary units) of simulations are taken as follows: $h_{0}=0.033, h_{1}=4, h_{2}=\frac{1}{4}, s_{i n}=10, s_{\text {ref }}=0.1, v_{m}=30, x_{0}=13$.

Table I. Time $t_{k}\left(v_{0}\right)$ of the singular arc strategy with $s_{0}=9$ and $k_{0}=0, k_{1}=10^{-4}, k_{2}=10^{-3}$.

\begin{tabular}{|c|c|c|c|c|c|c|c|c|}
\hline$v_{0}$ & 1 & 5 & 9 & 13 & 17 & 21 & 25 & 29 \\
\hline$t_{k_{0}}\left(v_{0}\right)$ & 310.1651 & 167.2351 & 117.1540 & 86.8963 & 65.5134 & 50.3793 & 37.8862 & 21.1462 \\
\hline$t_{k_{1}}\left(v_{0}\right)$ & 312.2648 & 168.1458 & 117.6926 & 87.2484 & 65.7451 & 50.4975 & 37.9331 & 21.1720 \\
\hline$t_{k_{2}}\left(v_{0}\right)$ & 332.5485 & 176.9264 & 122.7791 & 90.5304 & 67.8569 & 52.8893 & 38.3771 & 21.4075 \\
\hline
\end{tabular}

\section{CONCLUSIONS}

Thanks to a careful study of the switching functions provided by the Pontryagin maximum principle, we could generalize the results of $[3,11]$ to the case where the model includes mortality and hydrolysis of dead biomass. We can conclude that the optimal feedback control law, which is 
Table II. Time $t_{k}\left(v_{0}\right)$ of the singular arc strategy with $s_{0}=0.5$ and $k_{0}=0, k_{1}=10^{-4}, k_{2}=10^{-3}$.

\begin{tabular}{|c|c|c|c|c|c|c|c|c|}
\hline$v_{0}$ & 1 & 5 & 9 & 13 & 17 & 21 & 25 & 29 \\
\hline$t_{k_{0}}\left(v_{0}\right)$ & 314.8341 & 179.7194 & 135.5225 & 110.7341 & 93.7733 & 81.4263 & 71.9633 & 64.4641 \\
\hline$t_{k_{1}}\left(v_{0}\right)$ & 316.9834 & 180.6965 & 136.1483 & 111.1780 & 94.1084 & 81.6887 & 72.1743 & 64.6373 \\
\hline$t_{k_{2}}\left(v_{0}\right)$ & 338.4241 & 190.5360 & 142.9312 & 115.8383 & 97.7176 & 84.5983 & 74.5905 & 66.6930 \\
\hline
\end{tabular}

either bang-bang (for Monod type kinetics) or singular (for Haldane type kinetics), is robust in the presence of mortality and hydrolysis effects. In fact, when these parameters are not exactly known, the optimal synthesis obtained in [3] remains valid, and it can be implemented in the same way (see [1]). As a future work, we plan to study this optimal control problem when the hydrolysis acts on the system with delay or when it depends on the state of the system. Characterizing feedback controls in this setting is a more difficult question and needs further investigations.

\section{ACKNOWLEDGMENTS}

The authors are grateful to A. Rapaport, C. Lobry and J. Moreno for helpful discussions on the subject. The authors also thank the two anonymous referees as well as the Associate Editor for their very valuable comments which helped to improve the original version of the paper.

\section{REFERENCES}

1. Betancur MJ, Moreno JA, Moreno-Andrade I, Buitrón G. Practical optimal control of fed-batch bioreactors for the waste water treatment. Internat. J. Robust Nonlinear Control 2006; 16(3):173-190, doi:10.1002/rnc.1046.

2. Dochain D, Rapaport A. Minimal time control of fed-batch processes with growth functions having several maxima. IEEE Trans. Automat. Control 2011; 56(11):2671-2676, doi:10.1109/TAC.2011.2159424.

3. Moreno J. Optimal time control of bioreactors for the wastewater treatment. Optimal Control Appl. Methods 1999; 20(3):145-164, doi:10.1002/(SICI)1099-1514(199905/06)20:3〈145::AID-OCA651〉3.0.CO;2-J.

4. Hao X, Wang Q, Zhang X, Cao Y, van Mark Loosdrecht C. Experimental evaluation of decrease in bacterial activity due to cell death and activity decay in activated sludge. Water Research 2009; 43(14):3604 - 3612 .

5. Teng Z, Gao R, Rehim M, Wang K. Global behaviors of monod type chemostat model with nutrient recycling and impulsive input. J. Math. Chem. 2010; 47(1):276-294.

6. Bonnard A, Chyba M. Singular Trajectories and their role in Control Theory. Springer, SMAI, vol. 40, 2002.

7. Miele A. Application of Green's Theorem to the extremization of linear integrals. Symp. on Vehicle Systems Optimization, Garden City: New York, 1961.

8. Boscain U, Piccoli B. Optimal syntheses for control systems on 2-D manifolds, Mathématiques \& Applications (Berlin) [Mathematics \& Applications], vol. 43. Springer-Verlag: Berlin, 2004.

9. Monod J. Recherches sur la Croissance des Cultures Bactériennes. Hermann: Paris, 1942.

10. Smith HL, Waltman P. The Theory of the Chemostat, Dynamics of Microbial Competition, Cambridge Studies in Mathematical Biology, vol. 13. Cambridge University Press: Cambridge, 1995, doi:10.1017/CBO9780511530043.

11. Gajardo P, Ramírez C, Rapaport A. Minimal time sequential batch reactors with bounded and impulse controls for one or more species. SIAM J. Control Optim. 2008; 47(6):2827-2856, doi:10.1137/070695204.

12. Bayen T, Gajardo P, Mairet F. Optimal synthesis for the minimal time control problems of fed-batch processes for growth functions with two maxima. J. Optim. Theory Appl. published online, nov 2012; doi:DOI10.1007/ s10957-012-0225-0.

13. Bressan A, Rampazzo F. Impulsive control systems with commutative vector fields. Journal of Optim. Theory and Applicationss 1991; 71(2):67-83.

14. Miller B. The generalized solutions of nonlinear optimization problems with impulse control. SIAM J. Control and Optimization 1996; 34(4):1420-1440.

15. Bardi M, Capuzzo-Dolcetta I. Optimal control and viscosity solutions of Hamilton-Jacobi-Bellman equations. Systems \& Control: Foundations \& Applications, Birkhäuser Boston Inc.: Boston, MA, 1997, doi:10.1007/ 978-0-8176-4755-1. With appendices by Maurizio Falcone and Pierpaolo Soravia. 